ATMOSPHERIC NOISE 
ELEMENTS A series edited

by Stacy Alaimo and Nicole Starosielski 


\section{ATMOSPHERIC NOISE}

\section{THE INDEFINITE URBANISM}

\section{OF LOS ANGELES}

MARINA PETERSON

DUKE UNIVERSITY PRESS Durham and London 2021 
(C) 2021 Duke University Press

All rights reserved

Printed in the United States of America on acid-free paper $\infty$

Text design by Amy Ruth Buchanan

Cover design by Drew Sisk

Typeset in Chaparral Pro and Knockout by Copperline Book Services

Library of Congress Cataloging-in-Publication Data

Names: Peterson, Marina, author.

Title: Atmospheric noise : the indefinite urbanism of Los Angeles /

Marina Peterson.

Other titles: Elements (Duke University Press)

Description: Durham : Duke University Press, 2021. | Series:

Elements | Includes bibliographical references and index.

Identifiers: LCCN 2020035285 (print) | LCCN 2020035286 (ebook) |

ISBN 9781478010708 (hardcover) | ISBN 9781478011828 (paperback) |

ISBN 9781478013174 (ebook)

Subjects: LCsH: City noise-California-Los Angeles. | City sounds-

California-Los Angeles. | Noise pollution-California-Los Angeles. |

Sound-Environmental aspects-California-Los Angeles.

Classification: LCC TD893.3.C2 P48 2021 (print) |

LCC TD893·3.C2 (ebook) | DDC 363.7409794/94-dc23

LC record available at https://lccn.loc.gov/2020035285

LC ebook record available at https://lccn.loc.gov/2020035286 
for Cassius 
This page intentionally left blank 